\title{
Obesity, maternal smoking and SHBG in neonates
}

Swapna Dharashivkar ${ }^{1}$, Lawrence Wasser² ${ }^{2}$ Richard N. Baumgartner ${ }^{3}$, Jeffrey C. King ${ }^{4}$ and Stephen J. Winters ${ }^{1 *}$

\begin{abstract}
Background: Sex hormone binding globulin (SHBG), a glycoprotein produced by hepatocytes that transports testosterone and other steroids in plasma, is a marker for developing metabolic syndrome and T2DM. SHBG is present in umbilical cord blood where it may be epigenetically regulated. This study was conducted to investigate whether the fetal environment, based on maternal pre-pregnancy weight, pregnancy weight gain or smoking during pregnancy, influence SHBG in newborns.
\end{abstract}

Methods: Maternal and newborn characteristics and SHBG levels and other variables were measured in cord and day 2 heel-stick blood samples in 60 healthy full-term singleton babies (31 F, 29 M).

Results: SHBG levels varied nearly fivefold among male and female newborns and were unrelated to sex, neonatal adiposity, determined by the Ponderal index and skinfold thickness, nor TNF $\propto$ in cord blood. There were also no statistically significant associations between pre-pregnancy weight or pregnancy weight gain and newborn SHBG levels. However, cord blood SHBG was higher and insulin levels were lower when mothers were smokers, but normalized by day 2.

Discussion: While SHBG levels are low in obese children and adults, and portend the development of metabolic syndrome and T2DM, our study of healthy babies born to normal women, found no connection between maternal obesity or newborn adiposity and SHBG levels in newborns. Insofar as women who smoked during pregnancy were thinner and had lower cord blood insulin levels than nonsmokers, higher SHBG in their newborns at birth might have been due to insulin sensitivity, or perhaps to an effect of smoking on placental gene expression.

Conclusions: Factors other than maternal weight and pregnancy weight gain appear to be the major determinants of SHBG in newborns. Higher SHBG levels when mothers smoke during pregnancy may contribute to overweight beginning later in childhood. Whether newborn SHBG levels predict the development of overweight and metabolic syndrome remains to be determined.

Keywords: Sex hormone binding globulin, Maternal smoking, Obesity, Newborn

\section{Background}

Sex hormone binding-globulin (SHBG) is a circulating glycoprotein secreted predominantly by hepatocytes which binds certain sex steroids, including testosterone, dihydrotestosterone, and estradiol, and regulates their entry into target cells [1]. The SHBG gene, located on chromosome 17 (17p13-1p12), and its protein product

\footnotetext{
*Correspondence: sjwint01@louisville.edu

${ }^{1}$ Division of Endocrinology, Metabolism and Diabetes, University of Louisville, ACB-A3G11, 550 Jackson Street, Louisville, KY 40202, USA Full list of author information is available at the end of the article
}

are regulated by genetic factors, nutritional cues and various hormones. Notably, SHBG levels in children and adults are inversely related to adiposity and to insulin resistance [2]. SHBG levels are reduced in children born at low birth weight [3], in teenage children with the metabolic syndrome [4], and in pre-pubertal children whose parents have the metabolic syndrome [5]. Moreover, low levels of SHBG in adolescence predict adiposity and insulin resistance in adulthood [6, 7]. Thus there is increasing research on SHBG.

SHBG is present in cord blood where levels vary among individuals by as much as tenfold [8] but appear not to 
differ by sex $[9,10]$ or race [11]. Cord blood SHBG levels were inversely related to cord blood insulin in a combined population of healthy pregnant women and those with gestational diabetes, but mean levels did not differ between the two groups [12]. Cord blood SHBG levels were higher when pregnant women with PCOS were treated with metformin than with placebo [13]

The concept of fetal programming has been gaining momentum with increasing evidence that the intrauterine milieu influences disease development later in life [14]. Children born to obese mothers are more likely to become obese [15], and excessive maternal pregnancy weight gain results in higher birth weight infants, which, in turn, is associated with subsequent obesity and obesity-related negative health outcomes [16]. Cigarette smoking during pregnancy is associated with reduced fetal growth and an increased risk for intrauterine growth retardation $[17,18]$, with increased obesity as early as age 5 years [19], and with increased diabetes risk when babies reach adulthood [20]. In rats, prenatal nicotine exposure produced a marked hypertrophy in adipocytes, and a decrease in pancreatic islet size, along with glucose intolerance and insulin resistance in adulthood [21]. Newborn babies exposed in utero to tobacco smoke have increased cord blood concentrations of prolactin and growth hormone [22] and cortisol [23], but no studies have examined SHBG.

In this study, we sought to determine the impact of the intrauterine environment based on maternal pre-pregnancy weight and pregnancy weight gain, and maternal smoking status, as well as newborn adiposity on the variability in SHBG among neonates born to healthy mothers. Since SHBG is expressed in the placenta, and the mRNA is reduced in mothers with gestational diabetes [24], we studied SHBG in neonates on day 2 as well as in cord blood. This research begins to explore the overall hypothesis that SHBG levels in the fetus and newborn are partly under genetic control but are also regulated by the intrauterine environment, and may contribute to and predict the development of adiposity, metabolic syndrome and diabetes as children grow older.

\section{Methods}

We evaluated mothers without pre-eclampsia, diabetes or complications during pregnancy who gave birth to singleton babies born by vaginal delivery. Informed consent was obtained in accordance with a protocol approved by the Institutional Review Boards of the University of Louisville and Louisville University Hospital. Babies had an Apgar score of 7 or greater at $5 \mathrm{~min}$, and no apparent congenital anomalies. 66 mothers provided informed consent but data were complete for 60 women who gave birth to 31 girls and 29 boys. For 6 babies, blood samples were lost and not analyzed. There were 24 whites, 25 blacks, and 17 babies who were mixed race or other. Maternal pre-pregnancy weight was obtained by chart review or by recall. Gestational weight gain was calculated as the difference between the last weight measured before delivery and the pre-pregnancy weight. Placental weight was measured after removal of the umbilical cord and membranes. Mothers were classified as smokers if they answered 'yes' to the question, "have you smoked during your pregnancy?" All participants denied use of alcohol during pregnancy.

Birth weight $(\mathrm{gm})$ and length $(\mathrm{cm})$ were determined, and babies were re-examined on day 2 of age at which time the subscapular skin fold (SSF) and triceps skin fold (TSF) thickness were determined by a pediatrician (LW) using a Harpenden caliper. The Ponderal index, [(weight in $\left.\mathrm{gm}) \times 100 /(\text { length in } \mathrm{cm})^{3}\right]$ was calculated as a measure of infant adiposity.

Venous cord blood was collected from a clamped segment of the umbilical cord at delivery, and heel-stick blood samples were obtained during the physical examination on day 2. Glucose was measured using a glucometer (StatStrip blood glucose meter, Nova Biomedical, Waltham, MA, USA). Samples were centrifuged and stored at $-20{ }^{\circ} \mathrm{C}$ for assay. SHBG and insulin were measured using ELISA kits from Alpco (Salem, NH, USA). TNF $\propto$ was measured using the Quantikine HS ELISA Human TNF alpha assay (R and D Systems, Minneapolis, MN, USA).

Statistical analyses used t tests to compare group means and linear regression to evaluate associations of SHBG levels with continuous covariates. All analyses were conducted using STATA data analysis and statistical software (College Station, TX, USA). Effect modification by smoking was modeled using multiplicative interaction terms in regression analysis. Multiplicative interaction is the most common type of effect modifications; additive interaction, which is rarer, was not modeled.

\section{Results}

SHBG levels in cord blood ranged from 20.5 to $90.8 \mathrm{nmol} / \mathrm{L}$, and in the heel stick sample on day 2 from 15.2 to $80.3 \mathrm{nmol} / \mathrm{L}$. Overall there was no significant difference between the mean $( \pm \mathrm{SD})$ levels in boys $(45.1 \pm 18.9)$ and girls $(39.9 \pm 10.7 \mathrm{nmol} / \mathrm{L})$ in either cord blood or the samples taken on day $2(40.4 \pm 15.0 \mathrm{vs}$ $37.2 \pm 14.1 \mathrm{nmol} / \mathrm{L})$.

Results for women who smoked during pregnancy $(\mathrm{n}=14)$ and their babies are compared to nonsmokers $(\mathrm{n}=46)$ in Table 1 . Mothers who smoked were thinner, and their cord blood had lower insulin and higher SHBG levels than the non-smokers. The average birth weight of babies born to smokers was $93 \mathrm{~g}$ lower, which was $4.8 \%$ 
Table 1 Clinical characteristics and hormone values in women who smoked during pregnancy and nonsmokers, and their offspring

\begin{tabular}{|c|c|c|c|}
\hline Variable & Smokers $(n=14)$ & $\begin{array}{l}\text { Nonsmokers } \\
(n=46)\end{array}$ & p value \\
\hline $\begin{array}{l}\text { SHBG in cord blood } \\
(\mathrm{nmol} / \mathrm{L})\end{array}$ & $48.0 \pm 11.4$ & $39.7 \pm 13.7$ & 0.04 \\
\hline $\begin{array}{l}\text { Cord blood insulin } \\
(\mathrm{U} / \mathrm{L})\end{array}$ & $2.0 \pm 0.71$ & $3.1 \pm 1.94$ & 0.045 \\
\hline $\begin{array}{l}\text { Cord blood TNF } \propto \\
(\mathrm{pg} / \mathrm{mL})\end{array}$ & $6.92 \pm 2.54$ & $7.68 \pm 4.22$ & \\
\hline $\begin{array}{l}\text { Cord blood glucose } \\
(\mathrm{mmol} / \mathrm{L})^{\mathrm{a}}\end{array}$ & $6.32 \pm 1.10$ & $5.88 \pm 1.81$ & \\
\hline SHBG day 2 (nmol/L) & $40.4 \pm 20.0$ & $38.4 \pm 15.3$ & \\
\hline Birth weight (g) & $3032 \pm 402$ & $3184 \pm 401$ & \\
\hline Placenta weight (g) & $487 \pm 83$ & $480 \pm 111$ & \\
\hline Maternal age (years) & $25.7 \pm 4.7$ & $26.7 \pm 6.2$ & \\
\hline $\begin{array}{c}\text { Maternal pre-preg- } \\
\text { nancy wt (kg) }\end{array}$ & $60.9 \pm 13.0$ & $72.3 \pm 19.1$ & 0.03 \\
\hline $\begin{array}{l}\text { Pregnancy weight } \\
\text { gain }(\mathrm{kg})\end{array}$ & $14.3 \pm 7.4$ & $14.3 \pm 9.3$ & \\
\hline
\end{tabular}

less $(\mathrm{p}=\mathrm{NS})$, than for nonsmokers while placental weights were similar. One baby in the former group and two in the latter group were low birth weight $(<2500 \mathrm{~g})$. By day 2, SHBG levels in babies born to smokers declined to the levels of nonsmokers. Figure 1 shows the individual values for SHBG in smokers and non-smokers. In contrast to the babies born to smokers, SHBG levels in the newborns of non-smokers were similar in cord blood and on day 2 .

Figure 2 shows the relationship between cord blood insulin and SHBG. There was a statistically significant, inverse association of cord blood insulin with SHBG in smokers $\left(R^{2}=0.54 ; \mathrm{p}<0.003\right)$, but no association in nonsmokers $\left(R^{2}=0.03 ; p=0.26\right)$; hence, there was a statistically significant interaction between smoking and the association of cord insulin with SHBG $(\mathrm{p}=0.04)$. Multiple regression analysis suggested that the main effect of smoking on cord blood SHBG and the interaction of smoking with cord blood insulin were independent of pre-pregnancy weight, pregnancy weight gain, race, placental weight, birthweight and skinfold thickness. This finding suggests that maternal smoking modifies the relationship between insulin and SHBG in cord blood regardless of measures of maternal or infant body size and adiposity.

TNF $\propto$ was reported to suppress SHBG production in HepG2 hepatocarcinoma cells by decreasing the level of the transcription factor $\mathrm{HNF} 4 \propto$ [25]. In this study, cord blood TNF $\propto$ levels were not different between smokers
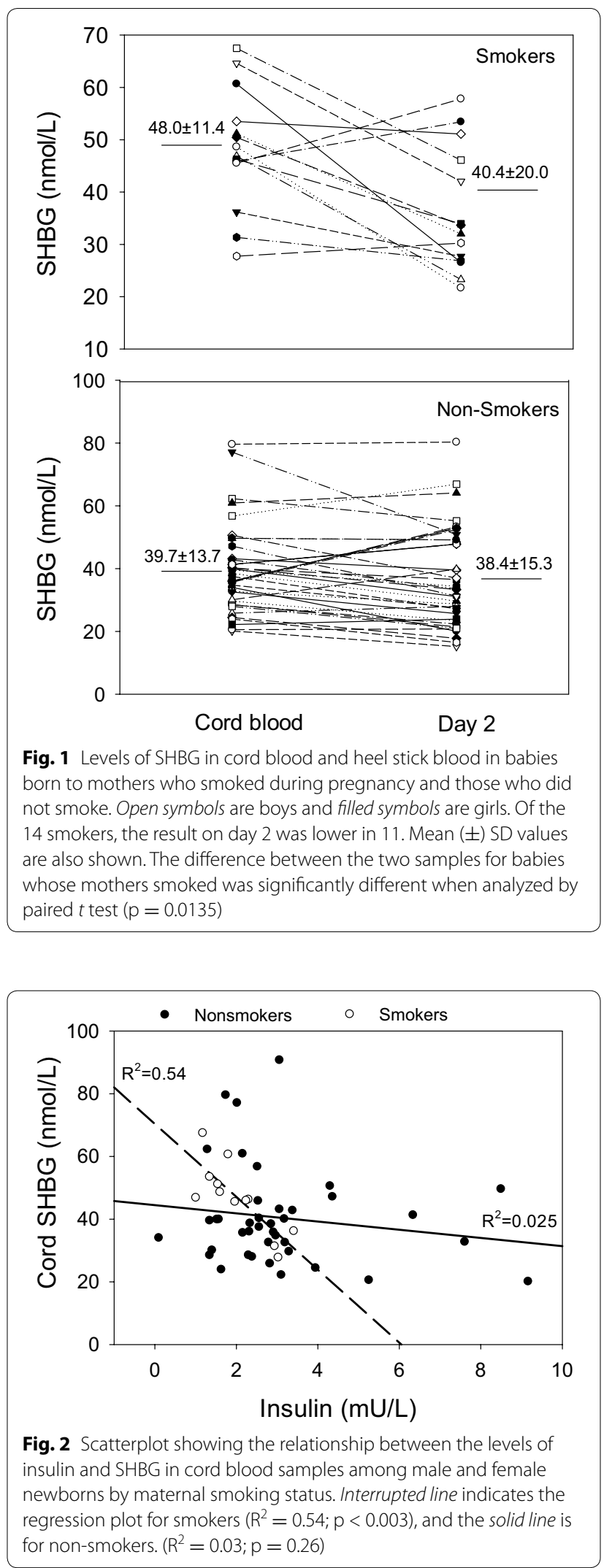
and nonsmokers, and were unrelated to cord blood SHBG $\left(R^{2}=0.03\right)$.

We next performed linear regression analysis to determine whether anthropometric variables were associated with SHBG (Fig. 3). Neither pre-pregnancy weight nor pregnancy weight gain was an important determinant of SHBG in cord blood. While there was a suggestion for an interaction between smoking and pregnancy weight gain with cord blood SHBG, it was not statistically significant $(p=0.30)$. Moreover, the apparent inverse association of pregnancy weight gain with cord blood SHBG in smokers $\left(R^{2}=0.20, p=0.11\right)$ was not altered by adjustment for insulin $\left(R^{2}=0.198, p=0.13\right)$. Similarly, the association of cord blood SHBG with pre-pregnancy weight $\left(\mathrm{R}^{2}=0.23, \mathrm{p}=0.08\right)$ was only slightly diminished by adjustment for insulin $\left(\mathrm{R}^{2}=0.19, \mathrm{p}=0.13\right)$. There was no association between pregnancy weight gain and day 2 heel-stick blood samples in either smokers or nonsmokers. In agreement with previous results [26], birth weight was positively related to placental weight $\left(R^{2}=0.35\right.$; $\mathrm{p}<0.01$; not shown). There was no significant relationship ( $p>0.05$ ) between SHBG on day 2 with either subscapular skinfold thickness, triceps skinfold thickness or flank skinfold thickness (not shown), birthweight, or the Ponderal index, a measure of fetal growth and nutrition (Fig. 4).

\section{Discussion}

The gestational period is a crucial time of growth, development and physiological change in the fetus. It is a window of opportunity for intervention via maternal nutrition and/or physical activity that may induce favorable changes in the in utero environment that will influence the lifelong risk for metabolic syndrome and T2DM.

The level of SHBG in plasma is known to be under genetic as well as hormonal and nutritional control. Because SHBG is inversely related to BMI beginning in childhood, and is a marker for insulin resistance and the risk for developing T2DM [1], we hypothesized that SHBG levels at birth might represent a biomarker of fetal

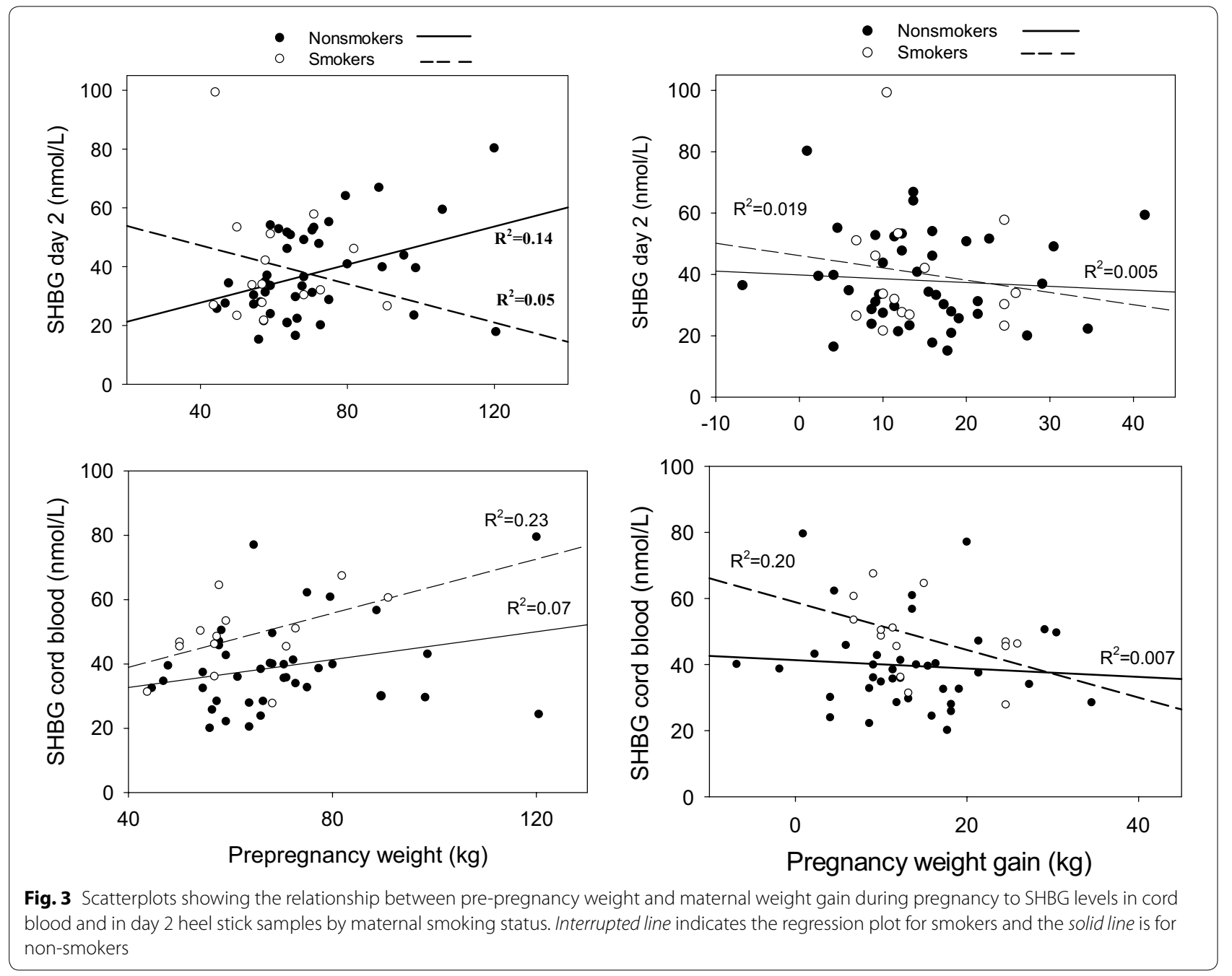




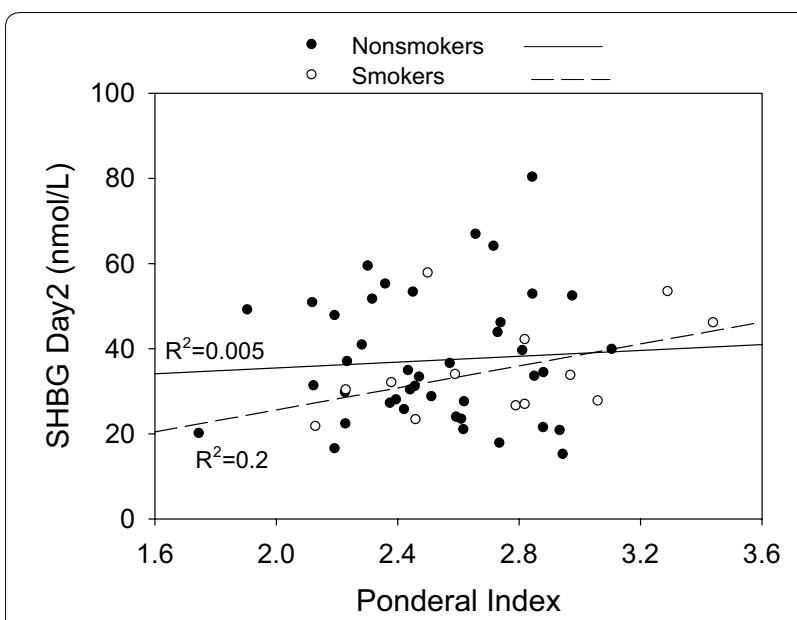

Fig. 4 Relationship between the Ponderal index and SHBG in newborn babies on day 2 by maternal smoking status

metabolic status, and might be related to the newborn's Ponderal index or skin fold thickness, or might correlate with maternal obesity or pregnancy weight gain. SHBG is expressed in the placenta which might contribute to the level in cord blood; therefore we show, for the first time, SHBG levels in heel-stick blood samples on day 2 as well as in cord blood samples. We recognize, however, that due to a long circulating half-life [27], a small amount of placental SHBG might remain in fetal blood on day 2 . SHBG levels in both cord blood and heel stick blood varied among individuals by fivefold, did not differ by sex, and were unrelated significantly to these anthropometric descriptors. In a previous study, SHBG levels were lower in babies born in Boston than in Shanghai where mothers had lower BMI and less pregnancy weight gain [28].

Instead, we discovered higher levels of SHBG in cord blood of maternal tobacco smokers that normalized by age 2 days. It is estimated that 4-25\% of women worldwide continue to smoke during pregnancy, and $24 \%$ of our new mothers viewed themselves as smokers. Maternal cigarette smoking is known to be pathogenic including increasing the risk for prematurity and low birth weight as well as obesity and type 2 diabetes in later life [18]. Mothers who smoked were thinner, and their cord blood insulin concentrations were lower, than for nonsmokers. BMI has been higher in maternal smokers than nonsmokers in some studies but not in others [29]. SHBG and insulin levels in cord blood were inversely related among mothers who smoked, but not among non-smokers, suggesting that smoking induces or enhances the relationship between insulin and SHBG. This finding has not been reported previously.

Higher SHBG levels in cord blood of smoking mothers may have resulted from indirect or direct effects of smoking on the fetal liver. To what extent higher SHBG levels in the cord blood of smokers was impacted by their thinness and lower insulin levels is not known. In a previous study, insulin and SHBG levels in cord blood were likewise inversely related among mothers with gestational diabetes [9]. While there is a substantial amount of data supporting an association between higher insulin levels and low levels of SHBG [30], no cellular mechanism has been established for this association, and more recent experiments using hepatocarcinoma cells have challenged the idea that insulin itself is a regulator of SHBG expression [31]. As much as $70 \%$ of the blood supply to the fetal liver is from the umbilical vein, resulting in exposure to high concentrations of xenochemicals from the maternal circulation. In this regard, maternal smoking was found to affect the expression level of a variety of genes in liver samples from fetuses from women undergoing second trimester elective pregnancy termination [32, 33]; however, no information was provided for SHBG.

Higher SHBG levels in cord blood of smokers might instead reflect SHBG of placental origin [24]. Placental DNA methylation patterns are altered with maternal smoking [34], and the expression level of placental genes related to coagulation and vasculogenesis is increased while the expression of cell adhesion-related genes is decreased; however, SHBG was not among the placental genes noted to be differentially expressed [35]. Although no studies have examined the effects of added insulin on placenta SHBG expression, SHBG mRNA levels were lower in placentas from hyperinsulinemic women with gestational diabetes [24]; so it is possible that elevated SHBG in cord blood in smokers in inverse relation to insulin levels was partly from placenta.

Several studies have linked higher maternal pre-pregnancy weight and greater gestational weight gain to adverse perinatal health outcomes including preterm birth, low-birth weight and macrosomia [36]. Moreover, maternal obesity has been associated with offspring with an increased risk for obesity lifelong as well as insulin resistance, hypertension and dyslipidemia, [37]. Higher circulating levels of inflammatory cytokines, insulin resistance and hyperinsulinemia, and dyslipidemia may cause permanent changes in the developing fetus. In one study of women from China, cord blood SHBG levels were lower among babies born to overweight mothers, most of whom had gestational diabetes [38]. In the current study, we found no inverse relationship between maternal obesity or weight gain during pregnancy with SHBG in the newborns of nondiabetic women, and no relationship between SHBG and neonatal markers of obesity including skin fold thickness and the Ponderal index, or to cord blood levels of the cytokine TNF $\propto$. Thus 
lower SHBG levels born to overweight diabetic women, if confirmed, might be due to diabetes rather than obesity. Moreover, genetic factors may contribute to the substantial variation in SHBG among newborns.

Limitations of this study include a relatively small sample size, especially for women who smoked during pregnancy, the reliance on recall for pre-pregnancy weight, and the broad definition of smoking during pregnancy. Denial of smoking would result in misclassification of smokers as non-smokers. Multiple hormonal and genetic factors are known to influence plasma SHBG levels in adults, but were not studied due to lack of sufficient sample volumes. Finally, intrahepatic fat was reported to be higher in infants born to obese mothers with gestational diabetes compared to infants of nondiabetic normal-weight mothers [39], and hepatic fat is known to be inversely associated with SHBG [40]; however, hepatic fat was not measured in the newborns in this study.

Maternal smoking is one of many factors associated with low birth weight and a subsequent increase in the risk for obesity in childhood and adult life. SHBG levels in low birth-weight boys were not statistically different from controls at age 2-3 months [41], but by age 8 years were lower in low birth weight girls [3], and low SHBG was found with obesity in both sexes by age 6-9 years [42] suggesting that the link between low SHBG and obesity is acquired at a young age. While low SHBG in older children and adults is a recognized marker of insulin resistance and hepatic steatosis, longitudinal studies are needed to determine whether SHBG levels at birth predict metabolic status later in life, and could be used as a marker for early interventions. Studies using human adult liver indicate that the major determinant of hepatic SHBG expression is the level of the transcription factor $H N F 4 \propto$, a key regulator of hepatocyte function [43]. Whether SHBG is an indicator of $H N F 4 \propto$ activity in fetal liver, or itself plays a direct role in fetal metabolism and subsequent metabolic health outcomes remains to be determined.

\section{Abbreviations \\ SHBG: sex hormone binding-globulin; PCOS: polycystic ovary syndrome; TNF $\propto$ : tumor necrosis factor $\propto$.}

\section{Authors' contributions}

SD obtained informed consent and study samples, helped examine babies and contributed to manuscript writing, LW examined all babies, RNB analyzed the data statistically, JCK supervised the activities of the labor and delivery suite, and SJW developed the research plan and contributed to data analysis and manuscript preparation. All authors read and approved the final manuscript.

\footnotetext{
Author details

1 Division of Endocrinology, Metabolism and Diabetes, University of Louisville, ACB-A3G1 1, 550 Jackson Street, Louisville, KY 40202, USA. ${ }^{2}$ Department of Pediatrics, University of Louisville, Louisville, KY 40202, USA. ${ }^{3}$ Department of Epidemiology and Population Health, University of Louisville, Louisville,
}

KY 40202, USA. ${ }^{4}$ Division of Maternal-Fetal Medicine, University of Louisville, Louisville, KY 40202, USA.

\section{Acknowledgements}

We thank the staffs of the Labor and Delivery Suite and the Newborn Nursery at University of Louisville Hospital for their help in conducting this study. We thank Mr. Dushan Ghooray for his patient assistance and careful technical help in the laboratory. SD was the recipient of an Endocrine Fellowship Award from AbbieVie. This research was supported in part by a gift from the Walter and Avis Jacobs Foundation. A portion of this research was presented at the 95th Annual Meeting of the US. Endocrine Society, San Francisco, CA, 2013, Abstract 5232.

\section{Competing interests}

The authors declare that they have no competing interests.

Received: 14 April 2016 Accepted: 10 July 2016

Published online: 26 July 2016

\section{References}

1. Hammond GL, Wu TS, Simard M. Evolving utility of sex hormone-binding globulin measurements in clinical medicine. Curr Opin Endocrinol Diabetes Obes. 2012;19:183-9.

2. Birkeland $\mathrm{Kl}$, Hanssen KF, Torjesen PA, Vaaler S. Level of sex hormonebinding globulin is positively correlated with insulin sensitivity in men with type 2 diabetes. J Clin Endocrinol Metab. 1993;76:275-8.

3. Ibanez L, Lopez-Bermejo A, Diaz M, de Zegher F. Catch-up growth in girls born small for gestational age precedes childhood progression to high adiposity. Fertil Steril. 2011;96:220-3.

4. Agirbasli M, Agaoglu NB, Orak N, et al. Sex hormones and metabolic syndrome in children and adolescents. Metab Clin Exp. 2009;58:1256-62.

5. Krishnasamy SS, Chang C, Wang C, et al. Sex hormone-binding globulin and the risk for metabolic syndrome in children of South Asian origin. Endo Prac. 2012;18:1-23.

6. Brand JS, van der Tweel I, Grobbee DE, et al. Testosterone, sex hormonebinding globulin and the metabolic syndrome: a systematic review and meta-analysis of observational studies. Int J Epidemiol. 2011;40:189-207.

7. Glueck CJ, Morrison JA, Daniels S, et al. Sex hormone-binding globulin, oligomenorrhea, polycystic ovary syndrome, and childhood insulin at age 14 years predict metabolic syndrome and class III obesity at age 24 years. J Pediatr. 2011;159(308-313):e302.

8. Savarese TM, Strohsnitter WC, Low HP, et al. Correlation of umbilical cord blood hormones and growth factors with stem cell potential: implications for the prenatal origin of breast cancer hypothesis. Breast Cancer Res. 2007;9:R29

9. Jin Z, Guan X, Gao H, et al. The change in sex hormone binding globulin and the influence by gestational diabetes mellitus in fetal period. Gynecol Endocrinol. 2009;25:647-52.

10. van de Beek C, Thijssen JH, Cohen-Kettenis PT, et al. Relationships between sex hormones assessed in amniotic fluid, and maternal and umbilical cord serum: what is the best source of information to investigate the effects of fetal hormonal exposure? Horm Behav. 2004;46:663-9.

11. Eichholzer M, Platz EA, Bienstock JL, et al. Racial variation in vitamin D cord blood concentration in white and black male neonates. Cancer Causes Control. 2013;24:91-8.

12. Simmons D. Interrelation between umbilical cord serum sex hormones, sex hormone-binding globulin, insulin-like growth factor I, and insulin in neonates from normal pregnancies and pregnancies complicated by diabetes. J Clin Endocrinol Metab. 1995;80:2217-21.

13. Vanky E, Stridsklev S, Heimstad R, et al. Metformin versus placebo from first trimester to delivery in polycystic ovary syndrome: a randomized, controlled multicenter study. J Clin Endocrinol Metab. 2010;95:E448-55.

14. Fall C, Osmond C. Commentary: the developmental origins of health and disease: an appreciation of the life and work of Professor David J.P. Barker, 1938-2013. Int J Epidemiol. 2013;42:1231-2.

15. Gillman MW, Rifas-Shiman SL, Kleinman K, et al. Developmental origins of childhood overweight: potential public health impact. Obesity. 2008;16:1651-6. 
16. Skilton MR, Siitonen N, Wurtz P, et al. High birth weight is associated with obesity and increased carotid wall thickness in young adults: the cardiovascular risk in young Finns study. Arterioscler Thromb Vasc Biol. 2014;34:1064-8.

17. Bernstein IM, Plociennik K, Stahle S, et al. Impact of maternal cigarette smoking on fetal growth and body composition. Am J Obstet Gynecol. 2000;183:883-6.

18. Mund M, Louwen F, Klingelhoefer D, Gerber A. Smoking and pregnancy - a review on the first major environmental risk factor of the unborn. Int J Environ Res Public Health. 2013;10:6485-99.

19. Mizutani T, Suzuki K, Kondo N, Yamagata Z. Association of maternal lifestyles including smoking during pregnancy with childhood obesity. Obesity. 2007;15:3133-9.

20. Lamerrill MA, Cirillo PM, Krigbaum NY, Cohn BA. The impact of prenatal parental tobacco smoking on risk of diabetes mellitus in middle-aged women. J Dev Orig Health Dis. 2015;6:1-8.

21. Somm E, Schwitzgebel VM, Vauthay DM, et al. Prenatal nicotine exposure alters early pancreatic islet and adipose tissue development with consequences on the control of body weight and glucose metabolism later in life. Endocrinology. 2008;149:6289-99.

22. Beratis NG, Varvarigou A, Makri M, Vagenakis AG. Prolactin, growth hormone and insulin-like growth factor-l in newborn children of smoking mothers. Clin Endocrinol. 1994;40:179-85.

23. Varvarigou AA, Petsali M, Vassilakos P, Beratis NG. Increased cortisol concentrations in the cord blood of newborns whose mothers smoked during pregnancy. J Perinat Med. 2006;34:466-70.

24. Sun $L$, Jin Z, Teng W, et al. SHBG in GDM maternal serum, placental tissues and umbilical cord serum expression changes and its significance. Diabetes Res Clin Pract. 2013;99:168-73.

25. Simo R, Barbosa-Desongles A, Saez-Lopez C, et al. Molecular mechanism of TNF $\propto$-induced down-regulation of SHBG expression. Mol Endocrinol. 2012;26:438-46.

26. Thame M, Osmond C, Bennett F, et al. Fetal growth is directly related to maternal anthropometry and placental volume. Eur J Clin Nutr. 2004;58:894-900.

27. Namkung PC, Stanczyk FZ, Cook MJ, et al. Half-life of plasma sex steroidbinding protein (SBP) in the primate. J Steroid Biochem. 1989;32:675-80.

28. Lagiou P, Samoli E, Hsieh CC, et al. Maternal and cord blood hormones in relation to birth size. Eur J Epidemiol. 2014;29:343-51.

29. Oken E, Levitan EB, Gillman MW. Maternal smoking during pregnancy and child overweight: systematic review and meta-analysis. Int J Obes. 2008;32:201-10.
30. Wallace IR, McKinley MC, Bell PM, Hunter SJ. Sex hormone binding globulin and insulin resistance. Clin Endocrinol. 2013;78:321-9.

31. Simo R, Saez-Lopez C, Barbosa-Desongles A, et al. Novel insights in SHBG regulation and clinical implications. Trends Endocrinol Metab. 2015;26:376-83.

32. O'Shaughnessy PJ, Monteiro A, Bhattacharya S, Fowler PA. Maternal smok ing and fetal sex significantly affect metabolic enzyme expression in the human fetal liver. J Clin Endocrinol Metab. 2011;96:2851-60.

33. Filis $P$, Nagrath $N$, Fraser $M$, et al. Maternal smoking dysregulates protein expression in second trimester human fetal livers in a sex-specific manner. J Clin Endocrinol Metab. 2015;100:861-70.

34. Nielsen $\mathrm{CH}$, Larsen $\mathrm{A}$, Nielsen AL. DNA methylation alterations in response to prenatal exposure of maternal cigarette smoking: A persistent epigenetic impact on health from maternal lifestyle. Arch Toxicol. 2014;90:231-45.

35. Votavova H, Dostalova Merkerova M, Krejcik Z, et al. Deregulation of gene expression induced by environmental tobacco smoke exposure in pregnancy. Nicotine Tob Res. 2012;14:1073-82.

36. Viswanathan M, Siega-Riz AM, Moos MK, et al. Outcomes of maternal weight gain. Evid Rep Technol Assess. 2008;16:1-223.

37. O'Reilly JR, Reynolds RM. The risk of maternal obesity to the long-term health of the offspring. Clin Endocrinol. 2013;78:9-16.

38. Liu B, Xu Y, Liang JM, et al. Intrauterine insulin resistance in fetuses of overweight mothers. J Obstet Gynaecol Res. 2013;39:132-8.

39. Brumbaugh DE, Tearse $P$, Cree-Green M, et al. Intrahepatic fat is increased in the neonatal offspring of obese women with gestational diabetes. J Pediatr. 2013;162(930-936):e931.

40. Shin JY, Kim SK, Lee MY, et al. Serum sex hormone-binding globulin levels are independently associated with nonalcoholic fatty liver disease in people with type 2 diabetes. Diabetes Res Clin Pract. 2011;94:156-62.

41. Sir-Petermann T, Maliqueo M, Codner E, et al. Early metabolic derangements in daughters of women with polycystic ovary syndrome. J Clin Endocrinol Metab. 2007:92:4637-42.

42. Gascon F, Valle M, Martos R, et al. Sex hormone-binding globulin as a marker for hyperinsulinemia and/or insulin resistance in obese children. Eur J Endocrinol. 2000;143:85-9.

43. Winters SJ, Gogineni J, Karegar M, et al. Sex hormone-binding globulin gene expression and insulin resistance. J Clin Endocrinol Metab. 2014;99:E2780-8

\section{Submit your next manuscript to BioMed Central and we will help you at every step:}

- We accept pre-submission inquiries

- Our selector tool helps you to find the most relevant journal

- We provide round the clock customer support

- Convenient online submission

- Thorough peer review

- Inclusion in PubMed and all major indexing services

- Maximum visibility for your research

Submit your manuscript at www.biomedcentral.com/submit 